\title{
Los procedimientos de un sistema de gestión de información: Un estudio de caso de la Universidad de Cienfuegos
}

\author{
Carlos Manuel Cañedo Iglesias \\ Mario Alberto Curbelo Hernández \\ Kirenia Núñez Chaviano \\ Raquel Zamora Fonseca \\ Universidad de Cienfuegos - Cuba
}

ESTUDOS / CASES

\begin{abstract}
Resumen
La investigación tiene como objetivo diseñar los procedimientos que identifiquen directamente los procesos claves o misionales de la biblioteca universitaria, en este caso, el desarrollo de colecciones o también llamado proceso de selección y adquisición. Se explicitan y describen los procedimientos para la mejora de la gestión de la información, mediante el empleo de herramientas universales (Diagrama SIPOC, diagrama de flujo estándar, y el procedimiento operativo, con el fin de identificar las necesidades de información, acceder a ellas, procesarlas y usarlas eficientemente de manera que en la formación del profesional se creen las competencias necesarias para su desempeño.
\end{abstract}

Palabras clave

Gestión por procesos; Desarrollo de colecciones; Selección y adquisición; Descripción de procedimientos; Bibliotecas universitarias; Universidad de Cienfuegos; Cuba

\section{The procedures of a management information system: A case report from the University of Cienfuegos}

\section{Abstract}

The research aims to design procedures that directly identify key or missionary processes of the University Library, in this case, the development of collections or also called selection and acquisition process. They are explicit and the procedures for improving the management of information, through the use of universal tools (diagram SIPOC, standard flow, and operating procedure diagram, in order to identify the information needs, access them, process them and use them efficiently so that in the formation of the professional competencies necessary for its performance are created).

Keywords

Process management; Development of collections; Selection and acquisition; Description of procedures; University libraries; University of Cienfuegos; Cuba

\section{Introducción}

La descripción de los procedimientos del sistema de gestión de la información en la biblioteca de la Universidad de Cienfuegos, sobre la base de aceptar como sistema más general el planteado en el - Macro proceso de gestión de los sistemas de información en bibliotecas--, estará compuesta por varios niveles de análisis, acorde con el enfoque 
en sistema. El primer nivel será ocupado precisamente por cada uno de los procesos claves o misionales y sus actividades principales.

La descripción de los procedimientos significa la aplicación de un grupo de herramientas gráficas y analíticas que permita describir el proceso relacionado, desde varios puntos de análisis, a fin de poder gestionarlos mejor en el futuro, adiestra la fuerza de trabajo según ciertas rutinas o estándares establecidos para la ejecución de las operaciones o actividades. En el caso específico de los procedimientos del sistema de gestión de la información de la biblioteca, se diseña el de selección y adquisición o desarrollo de colecciones el cual incluyen como herramientas importantes, los diagramas SIPOC, los diagramas de flujo estándar y el procedimiento operativo, herramienta que formará parte del quehacer del especialista en selección y adquisición en esta institución.

\section{Elaboración del diagrama SIPOC para el macro proceso:}

Para la comprensión de cada proceso es necesario conocer sus entradas para establecer los parámetros o requerimientos que esperan los clientes. EI SIPOC es una técnica que permite identificar cuáles son los suministradores del proceso, las entradas de cada suministrador al proceso, el proceso propiamente dicho, o sea, las etapas o fases del proceso, las salidas que emite el mismo y los clientes externos e internos que reciben estas salidas. En muchos estudios se identifican los requerimientos de calidad que desea el cliente para cada una de las salidas. Se utiliza fundamentalmente para identificar las variables de entradas y de salidas para un posterior análisis de estas y además a partir de las fases generales del proceso que se definen realizar análisis más detallados de estas fases posteriores en la gestión de procesos.

\begin{tabular}{|c|c|c|c|c|c|}
\hline Suministrador & Entrada & Proceso & Requerimientos & Salidas & Cliente \\
\hline $\begin{array}{l}\text { Almacenes de la UCF. } \\
\text { Organismos y personas } \\
\text { donantes. } \\
\text { Librerías e instituciones. }\end{array}$ & $\begin{array}{l}\text { Fuentes de información } \\
\text { documentales variadas (libros, } \\
\text { revistas, normas, periódicos, } \\
\text { mapas, } \\
\text { audiovisuales, documentos } \\
\text { sonoros) }\end{array}$ & & $\begin{array}{l}\text { Actualidad. } \\
\text { Disponibilidad. } \\
\text { Rapidez del servicio. } \\
\text { Amabilidad. } \\
\\
\text { Actualidad. } \\
\text { Disponibilidad. } \\
\text { Rapidez del servicio. } \\
\text { Amabilidad. }\end{array}$ & $\begin{array}{l}\text { Documentos de préstamo } \\
\text { externo. }\end{array}$ & $\begin{array}{l}\text { Estudiantes, profesores e } \\
\text { investigadores }\end{array}$ \\
\hline $\begin{array}{l}\text { Módulos bibliográficos de } \\
\text { la carreras, Módulos de } \\
\text { maestrías, estadísticas. } \\
\text { Tecnologías digitales } \\
\text { varias (PCs, red } \\
\text { alámbrica e inalámbrica, } \\
\text { videoteca, otros) }\end{array}$ & $\begin{array}{l}\text { Información sobre necesidades, } \\
\text { satisfacción } \\
\text { y comportamiento de usuarios. }\end{array}$ & $\begin{array}{l}\text { Proceso de } \\
\text { Información } \\
\text { (Biblioteca) }\end{array}$ & $\begin{array}{l}\text { Disponibilidad. } \\
\text { Trato amable y cordial. } \\
\text { Rapidez en el servicio. } \\
\text { Disponibilidad de hardware, } \\
\text { software necesario y medios } \\
\text { audiovisuales. } \\
\text { Formación multifacética del } \\
\text { especialista. }\end{array}$ & $\begin{array}{l}\text { Préstamo } \\
\text { Interbibliotecario. }\end{array}$ & $\begin{array}{l}\text { Estudiantes, profesores, } \\
\text { investigadores y miembros de } \\
\text { la comunidad científica y } \\
\text { académica }\end{array}$ \\
\hline $\begin{array}{l}\text { S.I.B. } \\
\text { Bases de datos. } \\
\text { Biblioteca Virtual. } \\
\text { Gestores bibliog. }\end{array}$ & $\begin{array}{l}\text { Tecnologías blandas (sistemas, } \\
\text { métodos, } \\
\text { software). }\end{array}$ & & $\begin{array}{l}\text { Formación de los especialistas. } \\
\text { Actualidad. } \\
\text { Actualidad de la } \\
\text { infnrmarión }\end{array}$ & Promoción de la lectura & $\begin{array}{l}\text { Estudiantes, profesores, } \\
\text { investigadores y miembros de } \\
\text { la comunidad cientifica y } \\
\text { académica }\end{array}$ \\
\hline
\end{tabular}

Figura 1. Diagrama SIPOC: Proceso de información. Fuente: Elaboración propia 


\section{Descripción del procedimiento de selección y adquisición.}

La selección es un proceso de análisis intelectual activo que tiene como base las políticas y procesos internos de cada biblioteca. La adquisición, en cambio, es un proceso administrativo, que estará basado en disponibilidad económica, descuentos, respuestas rápidas a proveedores; es un proceso hacia el exterior.

El estudio de la comunidad de usuarios y sus demandas ha de ser el factor que gobierna la selección. Hoy en día, algunos autores opinan que ya no debiera ser simplemente "selección y adquisición" sino incorporar el concepto de "Desarrollo de Colecciones", dentro del cual estaría incluido, siendo propósito construir una mejor colección para cubrir las necesidades de la comunidad.

Para conocimiento del proceso de selección y adquisición en la biblioteca de la Universidad de Cienfuegos (UCF) se muestra la ficha de proceso siguiente:

Es responsabilidad de la biblioteca identificar las necesidades e intereses de la comunidad. Las colecciones a formar deben se consistentes con las metas y objetivos de la institución. Para el procedimiento de selección es imprescindible la revisión de las listas básicas, el inventario del almacén principal de la UCF, módulos bibliográficos entregados por los coordinadores de las carreras; así como las solicitudes de los usuarios, la política de desarrollo de colecciones vigente para la selección y posterior adquisición de estas fuentes de información. EI SIB (Sistema Integro Bibliotecario es un producto informático elaborado en la Universidad de Cienfuegos), permite conocer la existencia de los distintos tipos de fuentes que se encuentran en la biblioteca y si no existen se realizara el análisis con los proveedores externos. Para adquirir estas fuentes es necesaria la aprobación de ATM (Departamento de Abastecimiento Técnico Material) y el Departamento de Contabilidad. El especialista del proceso de selección y adquisición debe convenir con el proveedor la forma de adquirir estos materiales; estas pueden ser: compra mediante fondos que se puede hacer directamente al editor o al librero, mediante la compra también se pueden adquirir las suscripciones que permiten la adquisición periódica de revistas y periódicos según lo establecido, el canje con otras escuelas o instituciones bibliotecarias y culturales; este afecta a los fondos duplicados o múltiples y las publicaciones realizadas por la institución por lo que se debe realizar una buena selección y como otra forma está la donación y legado. Al seleccionar las fuentes estas se trasladan hacia la biblioteca donde se inscriben en el libro de inscripción correspondiente donde son llenados los datos del título de la fuente, número de identificación (ID), forma de adquisición, precio y cantidad de ejemplares; estos mismos datos son plasmados en la primera pagina de la fuente en caso de que sea copia dura; si es adquirida información en formato digital esta es trasladada hacia ALFIN (Alfabetización Informacional) o a la biblioteca virtual. Por último se registran los mismos en el SIB para su posterior tratamiento en procesos técnicos.

Las necesidades de información llegan al sistema por diversas vías, pero la fundamental es mediante las solicitudes que hacen los futuros clientes del sistema, los cuales mediante un documento llamado Módulo Bibliográfico dan a conocer sus necesidades futuras y de esta forma llegan las solicitudes de información al puesto de selección y adquisición el cual se considera una entrada clave en este proceso. Esta entrada está conformada por las siguientes partes: Carreras, Maestrías, Investigadores (proyectos de investigación) y otros, pero el bibliotecario es quien tiene la visión de la colección global, por ello es el que está en mejores condiciones de definir una política de selección coherente y equilibrada. Su relación con el usuario, sus necesidades informativas, la utilización frecuente o esporádica de los documentos le permitirán decidir cuántos documentos, qué tipo de documentos, qué tipo de contenidos tendrán dichos documentos y cuáles serán los documentos concretos que formarán las colecciones.

En la Biblioteca de la UCF hay un especialista designado para cada carrera el cual se encarga de entregarle a la especialista del proceso de selección y adquisición el Módulo Bibliográfico, este debe estar en correspondencia con los diferentes planes de estudio que puede tener una misma carrera; en sus diferentes años, esto se realiza semestralmente para mantener una adecuada actualización y calidad del material que se pone a disposición del usuario; todo este proceso se realiza en los consejos de carrera y a partir de ahí el especialista del proceso se encarga de gestionar esta bibliografía con los diferentes proveedores. 
También como proveedores encontramos a los investigadores siendo estos un eslabón más para conocer las necesidades del cliente luego de concluido un servicio, porque es en ese preciso momento donde el cliente; sea profesor, estudiante o investigador en general, brinda su conformidad o no con el servicio prestado y en caso negativo, que otro ejemplar le hubiese gustado consultar para satisfacer sus expectativas y así poder enfocar

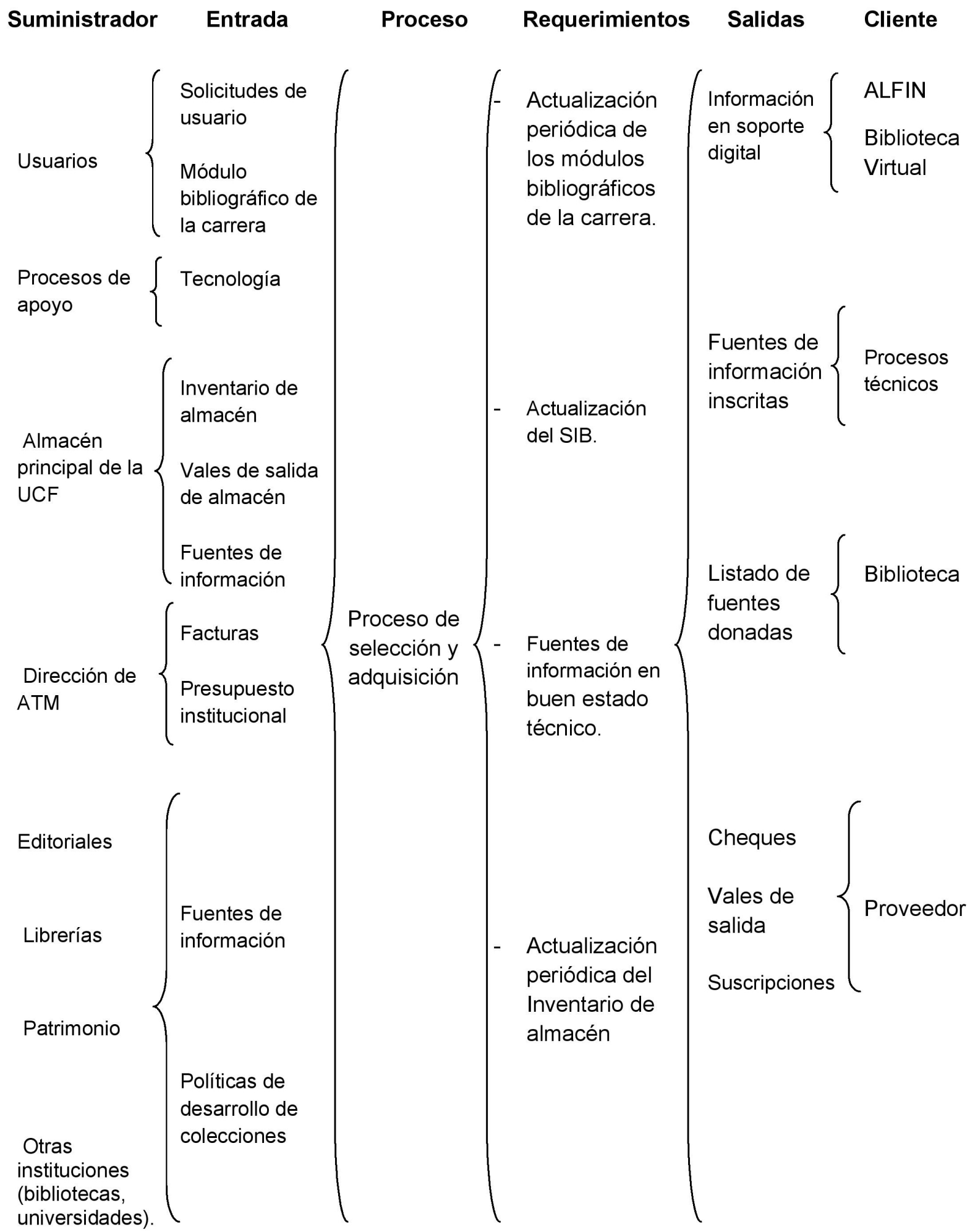


Biblios The procedures of an information management system 


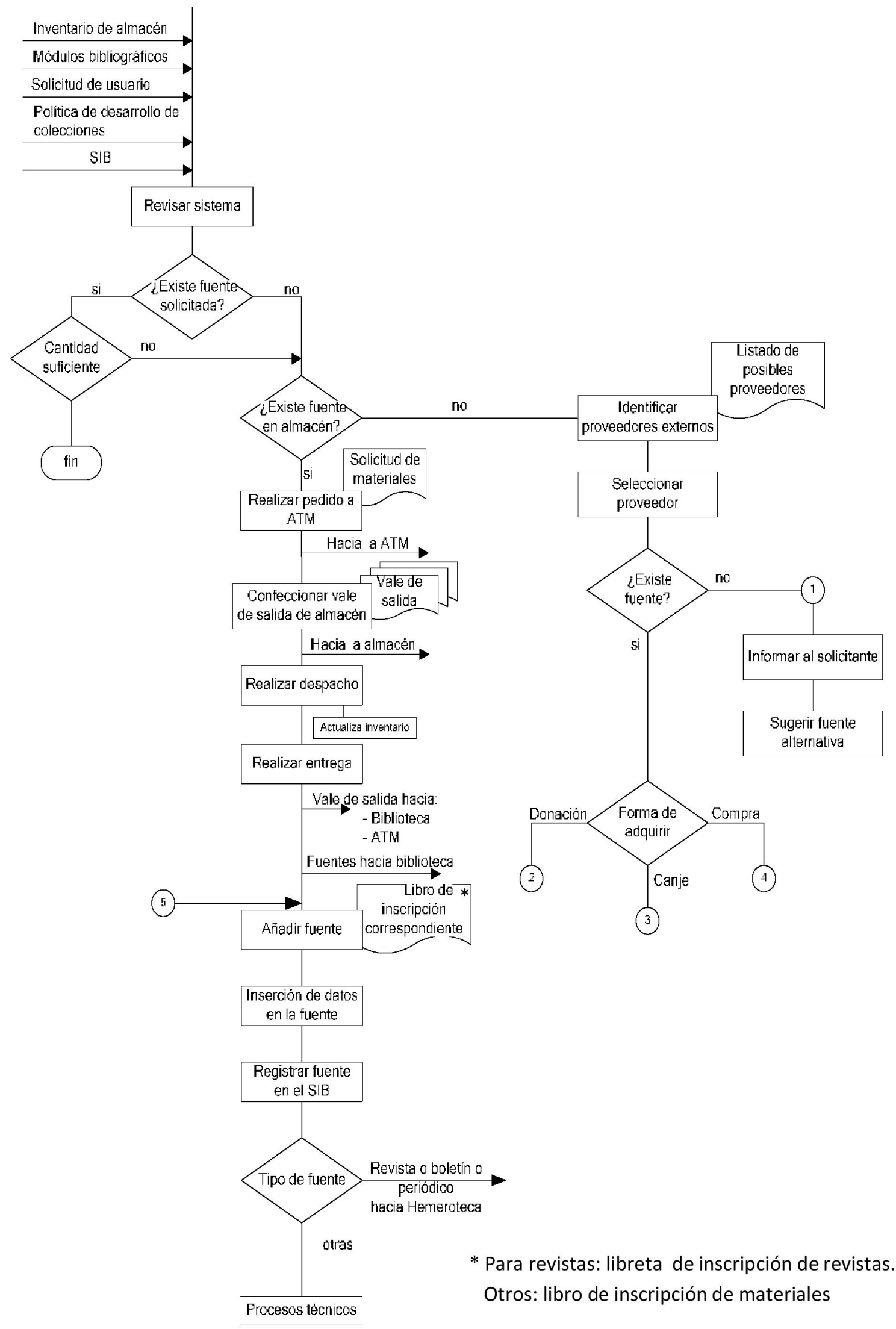

Figura 3. Diagrama de flujo: Proceso de información. Fuente: Elaboración propia 

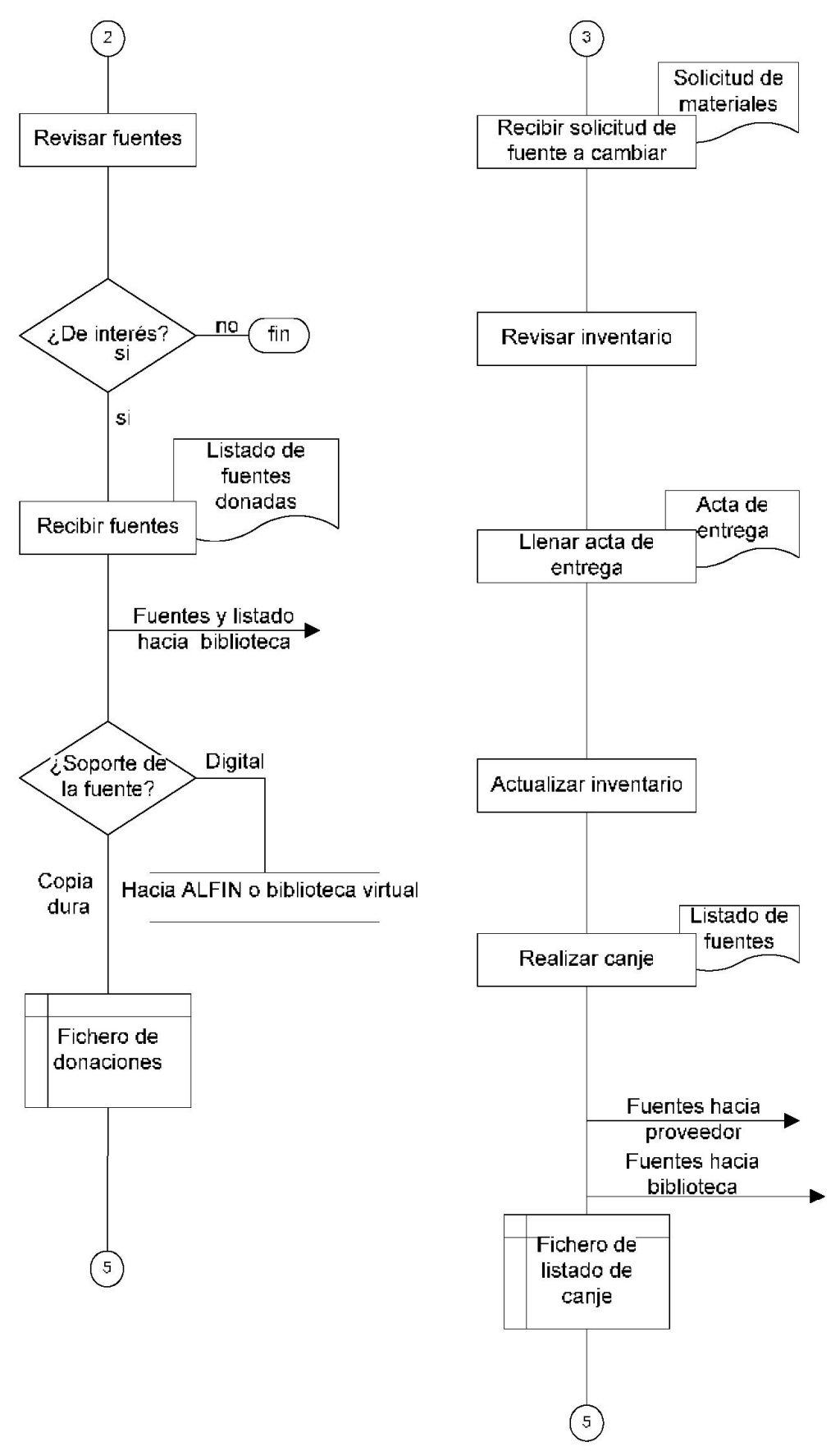

(4)
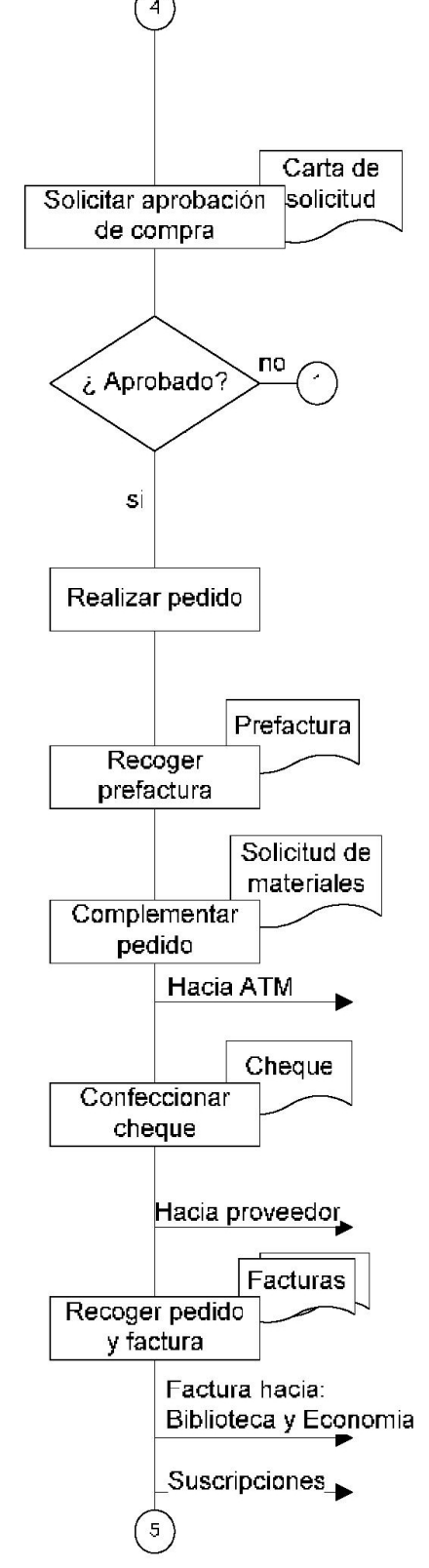

Figura 4. Diagrama de flujo estándar: Proceso de selección y adquisición: Fuente: Elaboración propia 


\begin{tabular}{|c|c|c|c|}
\hline Paso & Responsable & Actividad & $\begin{array}{l}\text { Documento de trabajo } \\
\text { clave }\end{array}$ \\
\hline 1 & $\begin{array}{l}\text { Especialista en Servicio, } \\
\text { análisis y procesamiento } \\
\text { de la información. }\end{array}$ & $\begin{array}{c}\text { Revisa sistema: a partir de las solicitudes de usuario y otras entradas se } \\
\text { analiza la existencia de las fuentes de información solicitadas en } \\
\text { biblioteca. Si existe la fuente, en las cantidades suficientes, finaliza el } \\
\text { proceso. Si no existe, se pasa al paso } 2 \text {. }\end{array}$ & SIB \\
\hline 2 & $\begin{array}{l}\text { Especialista en Servicio, } \\
\text { análisis y procesamiento } \\
\text { de la información. }\end{array}$ & $\begin{array}{l}\text { Revisa inventarios en almacén de la UCF. Si existe la fuente en } \\
\text { almacén, se pasa al paso 3.Si no existe, se pasa al paso } 8 .\end{array}$ & TEXSOL \\
\hline 3 & $\begin{array}{l}\text { Especialista en Servicio, } \\
\text { análisis y procesamiento } \\
\text { de la información. }\end{array}$ & $\begin{array}{l}\text { Realiza pedido a ATM: se elabora la solicitud de materiales con las } \\
\text { fuentes deseadas para realizar el movimiento interno de estas desde el } \\
\text { almacén hacia la biblioteca con el previo conocimiento de ATM. }\end{array}$ & Solicitud de materiales \\
\hline 4 & Especialista en ATM & $\begin{array}{c}\text { Confecciona vale de salida de almacén: constancia de las fuentes de } \\
\text { información a entregar a la biblioteca. }\end{array}$ & Vale de salida(3 copias) \\
\hline 5 & Almacén & Realiza despacho: se identifica la ubicación de las fuentes a entregar. & $\begin{array}{l}\text { Vale de salida(3 copias) } \\
\text { Tarjetas de estiba. }\end{array}$ \\
\hline 7 & Encargado de Almacén & Realiza entrega: Se entrega las fuentes solicitadas. & $\begin{array}{l}\text { Vale de salida(3 copias) } \\
\text { Tarjetas de estiba. }\end{array}$ \\
\hline 8 & $\begin{array}{l}\text { Especialista en Servicio, } \\
\text { análisis y procesamiento } \\
\text { de la información. }\end{array}$ & Identifica proveedores externos, de las fuentes solicitadas. & $\begin{array}{l}\text { Listado de posibles } \\
\text { proveedores. }\end{array}$ \\
\hline 9 & $\begin{array}{l}\text { Especialista en Servicio, } \\
\text { análisis y procesamiento } \\
\text { de la información. }\end{array}$ & $\begin{array}{l}\text { Selecciona proveedor más adecuado. Si la fuente está aún en falta, se } \\
\text { pasa al paso 10. Si esta es localizada, se pasa al paso } 11 .\end{array}$ & \\
\hline 10 & $\begin{array}{l}\text { Especialista en Servicio, } \\
\text { análisis y procesamiento } \\
\text { de la información. }\end{array}$ & Informa al solicitante y sugiere fuente alternativa. & \\
\hline 11 & $\begin{array}{l}\text { Especialista en Servicio, } \\
\text { análisis y procesamiento } \\
\text { de la información. }\end{array}$ & $\begin{array}{l}\text { Investiga la forma de adquisición directamente con el proveedor externo. } \\
\text { Si se trata de una donación, se pasa al paso } 12 \text {. Si es un canje se pasa } \\
\text { al paso16 y si se trata de una compra, se pasa al paso } 22 .\end{array}$ & \\
\hline 12 & $\begin{array}{l}\text { Especialista en Servicio, } \\
\text { análisis y procesamiento } \\
\text { de la información. }\end{array}$ & $\begin{array}{c}\text { Revisa físicamente las fuentes a canjear. Si no son de interés para el } \\
\text { usuario solicitante o para la biblioteca, concluye el proceso. Si las } \\
\text { fuentes son útiles, se pasa al próximo paso. }\end{array}$ & \\
\hline 13 & $\begin{array}{l}\text { Especialista en Servicio, } \\
\text { análisis y procesamiento } \\
\text { de la información. }\end{array}$ & $\begin{array}{c}\text { Recibe las fuentes donadas junto a un listado oficial con sus datos de } \\
\text { interés. }\end{array}$ & $\begin{array}{l}\text { Listado de fuentes } \\
\text { donadas. }\end{array}$ \\
\hline 14 & $\begin{array}{l}\text { Especialista en Servicio, } \\
\text { análisis y procesamiento } \\
\text { de la información. }\end{array}$ & $\begin{array}{l}\text { Envía las fuentes donadas a la sala de ALFIN, si estas son digitales. Si } \\
\text { la fuente es una copia dura, ejecuta el paso } 15 .\end{array}$ & \\
\hline 15 & $\begin{array}{l}\text { Especialista en Servicio, } \\
\text { análisis y procesamiento } \\
\text { de la información. }\end{array}$ & $\begin{array}{l}\text { Agrega el listado de fuentes donadas al fichero de donaciones. Después } \\
\text { ejecuta al paso } 28 .\end{array}$ & Fichero de donaciones \\
\hline 16 & $\begin{array}{c}\text { Especialista en Servicio, } \\
\text { análisis y procesamiento } \\
\text { de la información. }\end{array}$ & $\begin{array}{l}\text { Elabora y recibe solicitud de fuentes a canjear tanto para su recepción } \\
\qquad \text { como para su entrega. }\end{array}$ & $\begin{array}{l}\text { Solicitudes de } \\
\text { materiales }\end{array}$ \\
\hline 17 & $\begin{array}{c}\text { Especialista en Servicio, } \\
\text { análisis y procesamiento } \\
\text { de la información. }\end{array}$ & Revisa inventario de fuentes solicitadas. & SIB \\
\hline 18 & $\begin{array}{c}\text { Especialista en Servicio, } \\
\text { análisis y procesamiento } \\
\text { de la información. }\end{array}$ & Llena acta de entrega. & Acta de entrega. \\
\hline 19 & $\begin{array}{c}\text { Especialista en Servicio, } \\
\text { análisis y procesamiento } \\
\text { de la información. }\end{array}$ & Actualiza inventario en el SIB & SIB \\
\hline
\end{tabular}




\begin{tabular}{|c|c|c|c|}
\hline 20 & $\begin{array}{l}\text { Especialista en Servicio, } \\
\text { análisis y procesamiento } \\
\text { de la información. }\end{array}$ & Realiza canje. & Listado de fuentes. \\
\hline 21 & $\begin{array}{l}\text { Especialista en Servicio, } \\
\text { análisis y procesamiento } \\
\text { de la información. }\end{array}$ & $\begin{array}{l}\text { Agrega el listado de fuentes canjeadas al fichero de canjes. Después } \\
\text { ejecuta el paso } 28 .\end{array}$ & Fichero de canges. \\
\hline 22 & $\begin{array}{l}\text { Especialista en Servicio, } \\
\text { análisis y procesamiento } \\
\text { de la información. }\end{array}$ & $\begin{array}{l}\text { Solicita aprobación de compra. Si es rechazada la solicitud, culmina el } \\
\text { proceso. Si es aceptada la compra, se pasa al próximo paso. }\end{array}$ & $\begin{array}{l}\text { Carta de solicitud de } \\
\text { compra a ATM }\end{array}$ \\
\hline 23 & $\begin{array}{l}\text { Especialista en Servicio, } \\
\text { análisis y procesamiento } \\
\text { de la información. }\end{array}$ & Realiza el pedido al proveedor. & \\
\hline 24 & $\begin{array}{l}\text { Especialista en Servicio, } \\
\text { análisis y procesamiento } \\
\text { de la información. }\end{array}$ & Recoge prefactura del proveedor y envía a dirección de economía. & Prefactura. \\
\hline 25 & $\begin{array}{l}\text { Especialista en Servicio, } \\
\text { análisis y procesamiento } \\
\text { de la información. }\end{array}$ & Emite solicitud de materiales a ATM. & Solicitud de materiales \\
\hline 26 & Dirección de economía & Elabora cheque y envía a proveedor. & Cheque. \\
\hline 27 & $\begin{array}{l}\text { Especialista en Servicio, } \\
\text { análisis y procesamiento } \\
\text { de la información }\end{array}$ & $\begin{array}{l}\text { Recoge pedido y factura del proveedor. Una copia de factura hacia } \\
\text { economía y otra hacia biblioteca. Después ejecuta el paso } 28 .\end{array}$ & Factura. \\
\hline 28 & $\begin{array}{l}\text { Especialista en Servicio, } \\
\text { análisis y procesamiento } \\
\text { de la información. }\end{array}$ & $\begin{array}{l}\text { Añade fuente: se añaden las fuentes adquiridas llenando los campos } \\
\text { correspondientes al título de la fuente, número de identificación (ID), } \\
\text { forma de adquisición, precio y cantidad de ejemplares. }\end{array}$ & $\begin{array}{l}\text { Libro de inscripción } \\
\text { correspondiente }\end{array}$ \\
\hline 29 & $\begin{array}{l}\text { Especialista en Servicio, } \\
\text { análisis y procesamiento } \\
\text { de la información. }\end{array}$ & $\begin{array}{l}\text { Inserta datos en la fuente: se escriben los datos anteriores } \\
\text { correspondientes a la fuente en la primera hoja de esta. }\end{array}$ & \\
\hline 30 & $\begin{array}{l}\text { Especialista en Servicio, } \\
\text { análisis y procesamiento } \\
\text { de la información. }\end{array}$ & $\begin{array}{l}\text { Registra fuente en el SIB: se insertan los datos anteriores } \\
\text { correspondientes a cada fuente en el sistema para su posterior } \\
\text { procesamiento. Si la fuente es una revista, un boletín o un periódico, se } \\
\text { envía a la hemeroteca. Las demás fuentes, hacia procesos técnicos. }\end{array}$ & \\
\hline
\end{tabular}

Tabla 1. Procedimiento operativo. Proceso de selección y adquisición. Fuente: Elaboración propia

nuestro trabajo en el mejoramiento de deficiencias reales conocidas desde la fuente más confiable. Este método suele ser extremadamente eficiente pero se trabaja en base al error por lo que a pesar de su efectividad debe brindársele especial importancia al resto de las vías para adquirir información.

En cada biblioteca se adquieren obras de su especialidad que sirvan de apoyo a la investigación y a la docencia. La entrada de fuentes de información a la biblioteca cuenta con gran variedad de materiales: publicaciones periódicas (revistas, periódicos, boletines), colección de referencia impresa y material no librario (monografías de carácter histórico, científico, técnico), tesis, videos, CD y diapositivas).

La colección de referencia se integra por los documentos que sirven para una consulta rápida y puntual, como diccionarios, enciclopedias, directorios, biografías, bibliografías, catálogos, atlas, etc. También se obtienen fondos de apoyo a la investigación, que se trata del fondo dedicado a las labores de docencia de apoyo a la investigación. Los 
documentos que se incluyen aquí son: monografías especializadas, documentos de trabajo, tesis, normas y patentes e instrucciones técnicas. Se cuenta con fondos de extensión cultural los cuales incluyen: obras literarias como novelas, poemas, teatro, ensayos sobre temas de actualidad

La selección de los fondos no implica necesariamente su adquisición, ya que en la mayoría de las ocasiones existe la limitación presupuestaria. La biblioteca de la UCF no cuenta con un presupuesto designado para esta, es mediante la solicitud de materiales que la dirección de ATM hace entrega del presupuesto disponible para la adquisición de las fuentes de información.

La Figura 2 muestra la relación del proceso de selección y adquisición con el resto de los procesos, los proveedores y clientes del proceso. La secuencia de pasos a ejecutar por el Especialista en Servicio, análisis y procesamiento de la información en el proceso en cuestión se observan en el diagrama de flujo estándar que aparece en la Figura 4. El procedimiento operativo recoge lo descrito en los procesos anteriores y en un lenguaje para especialista en esta rama del saber, estableciendo una metodología paso a paso como se muestra en la Tabla 1.

\section{Conclusiones}

En el presente reporte se describen y establecen los algoritmos de los procedimientos del sistema de gestión de la información de la biblioteca de la Universidad de Cienfuegos "Carlos Rafael Rodríguez. Los componentes de los sistemas de información, en específico los que operan en las bibliotecas universitarias, los métodos y procedimientos sirven de elemento conector entre todos los demás. Los procedimientos en particular se encargan de coordinar las tecnologías tanto duras como blandas, y las personas involucradas de tal manera que se alcance transformar las entradas (fuentes de información), en salidas (productos y servicios), con un valor agregado tal que maximice la eficiencia de los procesos de servicios. El valor metodológico de los procedimientos propuestos radica en su utilidad para el entrenamiento de los actuales ocupantes de los cargos técnicos y especialistas de la biblioteca, para la planeación del trabajo y la capacitación de los mismos y como una herramienta más de ayuda a la formación de usuarios.

\section{Bibliografía}

Andrade Castro, Jesús Alberto. (2005). Discurso y uso de tecnologías de información: herramienta para la hegemonía del poder. Revista de Ciencias Sociales, 12(1), 89-104.

Andrea Ramírez., Paola. (2004). El opinador de la Profe. Retrieved from http://laprofe.blogspot.com/2004_02_22_laprofe_archive.html,2006-01-31

Artiles Visbal, Sara. (1998, diciembre). La reingeniería de procesos en los sistemas de información. Ciencias de la Información, 29(4), 27-34.

Bustelo Ruesta, Carlota. (2001). Tendencias en la gestion de la informacion, la documentacion y el conocimiento en las organizaciones. El Porfesional de la información, 10(12), 4-7.

Bustelo Ruesta, Carlota. (2001, March). Gestión del conocimiento y gestión de la información. Boletín del Instituto Andaluz de Patrimonio Histórico, VIII(34), 226-230.

Catálogo de la biblioteca. (2009). . Retrieved from http://www.worldlingo.com/ma/enwiki/es/Library catalog

Cerezal Mezquita J, Fiallo Rodríguez J. (2002). Los métodos científicos en las Investigaciones Pedagógicas. La Habana.

Cisneros Rodríguez, Inés. (2006). ¿Sociedad de la información, sociedad del conocimiento?: La educación como mediadora. Retrieved from http://tecnologiaedu.us.es/edutec/paginas/43.html

Dante Ponjuán, Gloria. (1999). Gestión, gestión de información, gestión del conocimiento...gestión del futuro. Ciencias de la Información, 30(3).

Enebral Fernández, José. (2006). Por la competencia en el manejo de la información. S.L.: S.N. 
García Rodríguez, Humberto. (2005). Las nuevas tecnologías. Retrieved from

http://www.gestiopolis.com/recursos/documentos/fulldocs/rrhh1/ntifrh.htm

La Biblioteca. (2009). . Retrieved from http://wikipedia.com

Massísimo, Angels. (2002). Evaluación de las colecciones de las bibliotecas universitarias. Anales de documentación, (5), 245-272.

Mercedes Caridad, Ana María Morales. (2006). Sociedad de la Información e inclusión digital en España: iniciativas y proyectos en marcha. España.

Morales Campos, Estela. (2008). La Sociedad de la Información en el Siglo XXI y la Biblioteca Universitaria. Retrieved from http://www.unesco.org/webworld/wirerpt/vers-web-htm

\section{Datos de los autores}

\section{Carlos Manuel Cañedo Iglesias}

Doctor en Ciencias Pedagógicas. Master en Educación. Profesor e Investigador del Centro de Estudio de Didáctica y Dirección de la Educación Superior (CEDDES). Universidad Carlos Rafael Rodríguez, Cienfuegos, Cuba.

ccanedo2010@yahoo.es

\section{Mario Alberto Curbelo Hernández}

Ingeniero Industrial. Master en Ciencias en Administración de Recursos Humanos. Profesor Auxiliar del Departamento de Ingeniería Industrial y de la Facultad de Ciencias Económicas y Empresariales. Universidad Carlos Rafael Rodríguez, Cienfuegos, Cuba.

mcurbelo@ucf.edu.cu

\section{Kirenia Núñez Chaviano}

Licenciada en Educación. Técnica de la Biblioteca de la Universidad Carlos Rafael Rodríguez, Cienfuegos, Cuba. knunez@ucf.edu.cu

\section{Raquel Zamora Fonseca}

Master en Educación. Es Especialista Principal de Análisis, Servicio y Procesamiento de la Información en la Biblioteca de la Universidad Carlos Rafael Rodríguez, Cienfuegos, Cuba.

rzamora@ucf.edu.cu

Recibido - Received: 2011-06-01

Aceptado - Accepted: 2012-03-30

\section{(cc) EY-NC-ND}

This work is licensed under a Creative Commons

Attribution-Noncommercial-No Derivative Works 3.0 United States License.

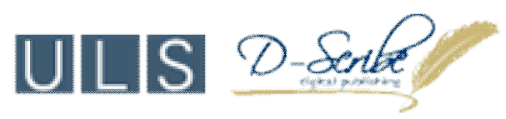

This journal is published by the University Library System of the University of Pittsburgh as part of its $\underline{D-S c r i b e}$ Digital Publishing Program and is cosponsored by the University of Pittsburgh Press. 